\section{EDUCATION}

Research, Innovation and Solutions on-line ${ }^{(0)}$
Electronic Journal of Research

in Educational Psychology

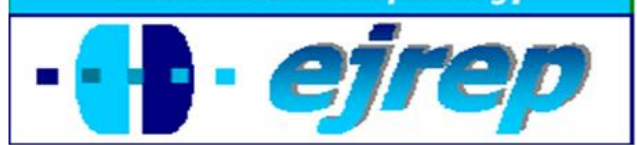

\title{
Relaciones entre la ansiedad estado y rasgo con la creatividad verbal y gráfica en alumnos de Educación Secundaria Obligatoria
}

\author{
Maria Teresa Sanz de Acedo Baquedano, \\ Maria Luisa Sanz de Acedo Lizarraga
}

España

Correspondencia: María Luisa Sanz de Acedo. Facultad de Psicología. Universidad Pública de Navarra. Spain. E-mail: mlsa@unavarra.es

(C) Education \& Psychology I+D+i and Editorial EOS (Spain) 


\section{Resumen}

Introducción. El objetivo de esta investigación fue examinar las relaciones existentes entre la ansiedad estado y rasgo y la creatividad verbal y gráfica así como la contribución de los tipos de ansiedad en la predicción de la creatividad en alumnos de Educación Secundaria Obligatoria.

Método. El estudio se realizó con 89 sujetos, de ambos sexos en edades comprendidas entre 12 y 14 años. Para ello, se aplicaron el Cuestionario de Autoevaluación Ansiedad Estado/Rasgo, formado por dos escalas independientes que miden situaciones transitorias y vivencias generales de tensión, y la Prueba de Imaginación Creativa, que evalúa dos tipos de creatividad con sus manifestaciones verbal y gráfica y la imaginación creativa o creatividad total.

Resultados. Los resultados mostraron una relación negativa y estadísticamente significativa entre los dos tipos de ansiedad y la creatividad verbal y gráfica, siendo más elevada y significativa con la ansiedad rasgo. Sin embargo, ni la ansiedad estado ni la ansiedad rasgo resultaron buenas predictoras de la creatividad verbal y gráfica y de la imaginación creativa, si bien la primera tuvo mayor capacidad predictiva que la segunda.

Conclusiones. Las conclusiones del estudio resultan interesantes a nivel de investigación pues aportan datos sobre las relaciones de la ansiedad con la creatividad.

Palabras clave: ansiedad estado, ansiedad rasgo, imaginación creativa, creatividad verbal y creatividad gráfica. 


\title{
Relationships between state and trait anxiety with verbal and graphic creativity in students in compulsory secondary education
}

\begin{abstract}
Introduction. The aim of this research was to examine the relationship between state and trait anxiety and verbal and graphic creativity and the contribution of the types of anxiety in predicting creativity in Compulsory Secondary Education students.

Method. The study was conducted with 89 subjects of both sexes aged between 12 and 14 years. For it, we applied the Questionnaire of Self-Assessment State/Trait Anxiety formed by two independent scales that measure transitory situations and general experiences of tension, and the Test of Creative Imagination, which evaluates two types of creativity with verbal and graphic manifestations and creative imagination or total creativity.
\end{abstract}

Results. The results showed a negative and statistically significant relationship between both types of anxiety and verbal and graphic creativity, and being significantly higher trait anxiety. However, neither state anxiety nor trait anxiety were good predictors of verbal and graphic creativity and creative imagination, although the first was more predictive than the second.

Discussion and conclusion. The findings are interesting in terms of research as they data on the relationship of anxiety with creativity.

Keywords: state anxiety, trait anxiety, creative imagination, verbal creativity and graphic creativity.

Received: 10/23/12 Initial acceptance: 10/50/12 Final acceptance: 11/20/12 


\section{Introducción}

La ansiedad es una emoción negativa que afecta a la supervivencia y el bienestar de la persona, pues se trata de un proceso que interviene en la adaptación y organización de su comportamiento. Por ser la ansiedad un concepto ambiguo, ha sido definida de muy diversas maneras, tales como un estado, un rasgo, un estímulo, un impulso, un motivo y una fuerza. Cattell y Scheier (1961) fueron los primeros en diferenciar ansiedad como estado y ansiedad como rasgo. Spielberger (1966) definió la ansiedad estado como la vivencia emocional transitoria caracterizada por la excitación y la ansiedad rasgo como la predisposición de un individuo para responder de una forma determinada.

La ansiedad puede mediar en determinados procesos cognitivos que intervienen en la ejecución creativa. Se sabe que afecta a la atención, la memoria de trabajo y la memoria a largo plazo (Fales et al., 2008; Tohill y Holyoak, 2000), incluso, correlaciona de manera negativa con la creatividad verbal y gráfica (Garaigordobil y Pérez, 2002). De hecho, algunas investigaciones señalan que la ansiedad influye en el pensamiento de las personas creativas haciéndolo menos eficiente. En consecuencia, la amplitud atencional de las personas creativas suele disminuir tanto a nivel perceptual, porque no pueden seleccionar una gran cantidad de estímulos y su búsqueda cognitiva está dirigida más bien por claves periféricas (Ansburg y Hill, 2003) como a nivel conceptual, ya que tienen dificultades en la producción de conexiones distintas (Mumford y Gustafson, 1988). Además, la ansiedad causa déficits en la memoria de trabajo de los sujetos creativos (Richards, French, Keogh, y Carter, 2000) porque en vez de utilizarla para combinar múltiples ideas y crear imágenes mentales, se ocupan de pensamientos recurrentes sobre la propia situación que genera ansiedad (Scott, Lonergan, y Mumford, 2005). Y lo mismo sucede con la memoria a largo plazo, la cual también se ve afectada por la ansiedad haciendo que las personas creativas tiendan a recordar la información congruente con su vivencia emocional (Eysenck y Mathews, 1987) sin dejar espacio para hacer asociaciones nuevas o, si las hacen, acceder a las mismas (Heilman, Nadeau, y Beversdorf, 2003).

La creatividad es un término de carácter multidimensional que genera muchas ideas (fluidez), variadas (flexibilidad), nuevas (originalidad) y detalladas (elaboración). Es, pues, una capacidad idónea para resolver problemas y desarrollar productos inéditos estimados como valiosos por la sociedad (Guilford, 1984; Sternberg y Lubart, 1999). Fruto de esta forma de pensar, puede analizarse desde cuatro perspectivas diferentes: un producto concreto, un 
proceso consciente e inconsciente, una persona con rasgos especiales y un ambiente social donde se manifiesta. Considerar la creatividad como la capacidad para producir algo novedoso, útil, de calidad e importante es la forma más generalizada de reconocer a una persona como creativa, ya que cuando un desenlace es original y útil resulta fácil inferir que el procedimiento que lo produjo fue creativo. También, puede ser entendida como un proceso de producción y comunicación de nuevas y significativas conexiones, para lo cual se utilizan distintas formas de pensamiento y se elaboran y seleccionan alternativas de acción. La creatividad, igualmente, es el resultado de la interacción de cualidades que la persona posee y que se proyectan en mayor o menor grado según las circunstancias (Sternberg y Lubart, 1995). La persona creativa es curiosa por las cosas que suceden en la vida, tiene confianza en sus posibilidades, tolera la ambigüedad, es flexible y extrovertida, vive en armonía con sus emociones al mismo tiempo que es sensible a las emociones de los demás y, por último, acepta el riesgo cuando desea dar cierto salto mental en su producción (Barron y Harrington, 1981; Kashdan y Fincham, 2002; Sternberg y Lubart, 1993). El ambiente, por supuesto, tiene su influencia en la creatividad, puesto que ayuda a maximizar la ejecución creativa fomentando la inspiración y evaluando los resultados.

La relación entre ansiedad y creatividad se ha analizado en numerosos estudios con resultados contradictorios, ya que en algunas ocasiones la ansiedad ha demostrado tener conexiones negativas (Matthews, 1986), positivas (Swanner, 1985) o no significativas (Kaufmann y Vosburg, 1997) con el rendimiento en tareas creativas. También, las investigaciones sugieren que la ansiedad tiene muchos efectos debilitadores, por ejemplo, puede bloquear el aprendizaje complejo (Eysenck, Derakshan, Santos, y Calvo, 2007). De hecho, varios modelos teóricos, tales como el del procesamiento dual (Kinsbourne y Hicks, 1978), el de la interferencia cognitiva (Tobias, 1985) y el de inhibición de la conducta (Flaherty, 2005), señalan las consecuencias perjudiciales de la ansiedad en la creatividad, caso de los individuos ansiosos que parecen pensar de forma ineficiente (Eysenck et al., 2007). La ansiedad también tiene algunos efectos facilitadores, como pueden ser la motivación al esfuerzo en la resolución de una tarea (Jones, Hanton, y Swain, 1994) y el aumento de la adopción de metas de logro (Elliot y McGregor, 1999).

Cabe mencionar, finalmente, algún trabajo que ha profundizado en la influencia de la ansiedad - estado o rasgo- en la creatividad verbal y gráfica. Concretamente, se ha observado que la ansiedad rasgo influye de forma más negativa que la ansiedad estado en la ejecución 
creativa y que la ansiedad en general interviene más negativamente en la creatividad verbal que en la gráfica (Byron y Khazanchi, 2011). Sin embargo, todavía son necesarias más investigaciones que estudien conjuntamente ambos tipos de ansiedad y de creatividad empleando muestras escolares en las que las dos variables pueden ser ya objeto de identificación y evaluación.

Por ello, teniendo como referencia los resultados comentados, los cuales son un tanto confusos, la presente investigación realizada en alumnos de Educación Secundaria Obligatoria (ESO) tuvo como objetivos: 1) analizar las relaciones existentes entre ansiedad estado y rasgo y creatividad verbal y gráfica y 2) estimar la contribución de estos dos tipos de ansiedad en la predicción de la ejecución creativa a través de palabras y dibujos.

\section{Método}

\section{Participantes}

El número de sujetos que participó en el estudio fue de 89, de ambos sexos (48 chicos y 41 chicas), con una edad comprendida entre 12 y 14 años $(M=12,91$; DT = 0,67) y pertenecientes a un centro educativo público y otro privado, de la ciudad de Pamplona, situados en uno de sus barrios de clase social media-alta. La evaluación se realizó dentro del horario escolar, la participación de los alumnos fue voluntaria aunque contó con la autorización previa de los padres y el clima de clase fue de curiosidad, aceptación y colaboración.

\section{Instrumentos de medida}

Para evaluar las variables objeto de estudio se utilizaron los instrumentos que se describen a continuación:

Cuestionario de Autoevaluación Ansiedad Estado/Rasgo (STAIC). Este cuestionario, diseñado por Spielberger (2001), se concibió como una herramienta de investigación en el estudio de la ansiedad en escolares de entre 9 y 15 años. Está formado por dos escalas independientes de autoevaluación para medir dos aspectos de la misma. Por un lado, la escala de ansiedad-estado, que comprende 20 elementos con los que los sujetos expresan "cómo se sienten en un momento determinado" e intenta evaluar sentimientos subjetivos de aprensión, tensión y preocupación que fluctúan en intensidad con el tiempo; y por otro lado, la escala de 
ansiedad-rasgo, que también contiene 20 elementos con los que el sujeto puede indicar "cómo se siente en general", pretende medir las diferencias relativamente estables de propensión a la ansiedad entre los sujetos con tendencia a mostrar estados de ansiedad. Este cuestionario exige un nivel adecuado de capacidad lectora para comprender los contenidos verbales de los estímulos y puede aplicarse de forma individual o colectiva en un tiempo aproximado de 20 minutos. Presenta una fiabilidad alta tanto para la ansiedad rasgo $(\alpha=0,93)$ como para la ansiedad estado $(\alpha=0,87)$. En cuanto a la validez concurrente se observa que la escala de ansiedad-rasgo correlaciona de un modo positivo y significativo $(r=0,75)$ con la prueba Children's Manifiest Anxiety Scale for Children (Castaneda, McCandless, y Palermo, 1956).

Prueba de Imaginación Creativa (PIC). Esta prueba fue diseñada por Artola, Ancillo, Mosteiro, y Barraca (2004) para evaluar la creatividad verbal y la creatividad gráfica. Consta de cuatro juegos, los tres primeros evalúan la creatividad verbal y el cuarto la creatividad gráfica y puede administrarse individual o colectivamente en aproximadamente 45 minutos. En el juego 1, a partir de una situación que aparece reflejada en un dibujo (un niño abriendo un cofre), el sujeto tiene que escribir todo aquello que pudiera estar ocurriendo en la escena; en el juego 2, que se trata de una adaptación del Test de Guilford ("Usos de un ladrillo"), el sujeto tiene que responder sobre los usos posibles de un tubo de goma; en el juego 3, se plantea una situación inverosímil con el objetivo de que el alumno exprese ideas poco convencionales que probablemente el sujeto no se atrevería a expresar en situaciones más serias y, por último, en el juego 4, inspirado en algunos ítems del Test de Torrance (Torrance, 1974), el sujeto tiene que completar cuatro dibujos a partir de unos trazos dados y poner un título a cada uno de ellos. La prueba permite obtener tres puntuaciones: a) la creatividad verbal compuesta de fluidez, flexibilidad y originalidad narrativas; b) la creatividad gráfica integrada por originalidad, elaboración, título y detalles especiales y c) una puntuación total que indica la imaginación o fantasía creativa que el niño ha demostrado en la realización de las tareas. Presenta una fiabilidad altamente satisfactoria $(\alpha=0,87)$ y una validez concurrente positiva y significativa con el Test de Inteligencia Creativa (Corbalán et al., 2003) tanto en la creatividad verbal $(r=0,43)$ como en la creatividad gráfica $(r=0,21)$ y en la imaginación creativa o creatividad total $(r=0,44)$. En la muestra estudiada la fiabildad obtenida fue para la creatividad verbal de $r=0,85$, la creatividad gráfica de $r=0,79$ y la imaginación creativa de $r=0,80$. 


\section{Procedimiento}

Las pruebas se realizaron en una única sesión que duró hora y media. En primer lugar, se administró el Cuestionario de Autoevaluación Ansiedad Estado/Rasgo (STAIC). Se emplearon 25 minutos, cinco de ellos para leer las instrucciones y aclarar cualquier duda referente al vocabulario y a la forma de contestar el cuestionario y 20 minutos para responder a las dos escalas que comprende el test. En segundo lugar, se aplicó la Prueba de Imaginación Creativa (PIC), con una duración total de 45 minutos, cinco de ellos para explicar las instrucciones redactadas en cada uno de los juegos y aclarar las posibles dudas y 40 minutos para realizar el total de los juegos (10 minutos para cada uno de los cuatro juegos).

\section{Análisis de datos}

El análisis de los datos se realizó utilizando los coeficientes de correlación de Pearson y el modelo de regresión lineal simple, ambos calculados con el software SPSS 19.0.

\section{Resultados}

Los resultados de este estudio se muestran en dos apartados donde se comentan las correlaciones entre los distintos tipos de ansiedad y creatividad y las estimaciones de la contribución de los tipos de ansiedad en la predicción de los resultados creativos.

\section{Correlaciones}

Con la finalidad de clarificar las relaciones existentes entre la ansiedad estado y rasgo, la creatividad verbal y gráfica con sus respectivos índices y la imaginación creativa, se hallaron los coeficientes de correlación de Pearson con las puntuaciones directas obtenidas por los sujetos en las pruebas administradas (Tabla 1). 
Tabla 1. Correlaciones entre la ansiedad estado y rasgo, los índices de la creatividad verbal y gráfica y la imaginación creativa. $(\mathrm{N}=89)$

\begin{tabular}{|c|c|c|c|c|c|c|c|c|c|c|c|}
\hline & 1 & 2 & 3 & 4 & 5 & 6 & 7 & 8 & 9 & 10 & 11 \\
\hline 1- AEs & -- & & & & & & & & & & \\
\hline 2- ARg & $0,87 * *$ & -- & & & & & & & & & \\
\hline 3- Flu & $-0,33 * *$ & $-0,50 * *$ & -- & & & & & & & & \\
\hline 4- Fle & $-0,33 * *$ & $-0,50 * *$ & $0,89 * *$ & -- & & & & & & & \\
\hline $5-\mathrm{OgV}$ & $-0,29 * *$ & $-0,48 * *$ & $0,64 * *$ & $0,69 * *$ & -- & & & & & & \\
\hline 6- CV & $-0,35 * *$ & $-0,53 * *$ & $0,95 * *$ & $0,96 * *$ & $0,80 * *$ & -- & & & & & \\
\hline 7- OgG & $-0,30 * *$ & $-0,32 * *$ & 0,12 & 0,15 & $0,22 *$ & 0,17 & -- & & & & \\
\hline 8- Elb & $-0,23^{*}$ & $-0,31 * *$ & 0,15 & 0,17 & 0,18 & 0,18 & $0,73 * *$ & -- & & & \\
\hline 9- Tit & $-0,32 * *$ & $-0,46 * *$ & 0,10 & 0,07 & 0,16 & 0,11 & $0,31 * *$ & $0,35^{* *}$ & -- & & \\
\hline 10- Des & $-0,09$ & $-0,06$ & $-0,01$ & $-0,00$ & 0,11 & 0,02 & $0,66 * *$ & $0,46 * *$ & 0,19 & -- & \\
\hline $11-\mathrm{CG}$ & $-0,34 * *$ & $-0,43 * *$ & 0,13 & 0,14 & $0,23 *$ & 0,17 & $0,85 * *$ & $0,78 * *$ & $0,71 * *$ & $0,66^{* *}$ & -- \\
\hline 12- IC & $-0,40 * *$ & $-0,59 * *$ & $0,93 * *$ & $0,94 * *$ & $0,81 * *$ & $0,98 * *$ & $0,32 * *$ & $0,33 * *$ & $0,24 *$ & 0,15 & $0,35 * *$ \\
\hline
\end{tabular}

Nota. AEs = Ansiedad Estado $;$ ARg = Ansiedad Rasgo $;$ Flu = Fluidez $;$ Fle = Flexibilidad $;$ OgV= Originalidad Verbal $;$ CV = Creatividad Verbal; OgG = Originalidad Gráfica; Elb = Elaboración; Tit = Título; Des = Detalles Especiales; CG = Creatividad Gráfica; IC = Imaginación Creativa.

$* p<.05 \quad * * p<.01$

La correlación obtenida entre ansiedad estado y rasgo ha sido positiva, alta y estadísticamente significativa $(r=0,87, p<.001)$ mientras que la encontrada entre la creatividad verbal y gráfica ha sido también positiva pero muy baja y no significativa $(r=0,17, p<.11)$. Si analizamos las correlaciones alcanzadas entre ansiedad estado y rasgo con cada uno de los índices que componen la creatividad verbal (fluidez, flexibilidad y originalidad narrativa) y la creatividad gráfica (originalidad, elaboración, título y detalles especiales), observamos que tanto las puntuaciones de dichos índices como las de la creatividad verbal, la creatividad gráfica y la imaginación creativa correlacionan con los dos tipos de ansiedad de forma negativa y estadísticamente significativa, a excepción del índice detalles especiales que aún siendo negativo no fue estadísticamente significativo ni con ansiedad estado $(r=-0,09, p<0,50)$, ni con ansiedad rasgo $(r=-0,06, p<.57)$. Además, todos los índices que integran los dos tipos de creatividad correlacionan con la ansiedad rasgo de manera más elevada, negativa y significativa que con la ansiedad estado. Igual sucede con las correlaciones entre creatividad verbal $(r=-0,53, p<.001)$, gráfica $(r=-0,43, p<.001)$ e imaginación creativa $(r=-0,59, p<.001)$ y ansiedad rasgo que son más altas si las comparamos con las obtenidas entre creatividad verbal $(r=-0,35, p<.001)$, gráfica $(r=-0,34, p<.001)$ e imaginación creativa $(r=-0,40, p<$ .001) con ansiedad estado.

Otro dato a destacar es que las correlaciones entre los dos tipos de creatividad e imaginación creativa son ambas positivas y estadísticamente significativas; sin embargo, las pun- 
tuaciones obtenidas son mucho más elevadas con la creatividad verbal $(r=0,98, p<.001)$ que con la gráfica $(r=0,35, p<.001)$. Asimismo ocurre con sus respectivos índices: fluidez $(r=$ $0,93, p<.001)$, flexibilidad $(r=0,94, p<.001)$ y originalidad narrativa $(r=0,81, p<.001)$ de la creatividad verbal y originalidad gráfica $(r=0,32, p<.001)$, elaboración $(r=0,33, p<.001)$, título $(r=0,24, p<.02)$ y detalles especiales $(r=0,15, p<.17)$ de la creatividad gráfica.

Finalmente, puede afirmarse que la ansiedad y la creatividad se relacionan en sentido inverso, es decir, cuando una aumenta la otra disminuyen, y viceversa. Así, los sujetos más ansiosos se mostraron menos creativos y los creativos, menos ansiosos.

Variables predictivas de la creatividad

Se realizaron dos análisis de regresión lineal simple. En el primero se escogió la ansiedad estado como predictor de la creatividad verbal y gráfica con sus respectivos índices y de la imaginación creativa (Tabla 2) y en el segundo, se eligió a la ansiedad rasgo como predictor de las mismas variables (Tabla 3 ).

Tabla 2. Análisis de regresión lineal simple de la ansiedad estado para predecir su relación con los índices de la creatividad verbal y gráfica y la imaginación creativa. $(\mathbf{N}=89)$

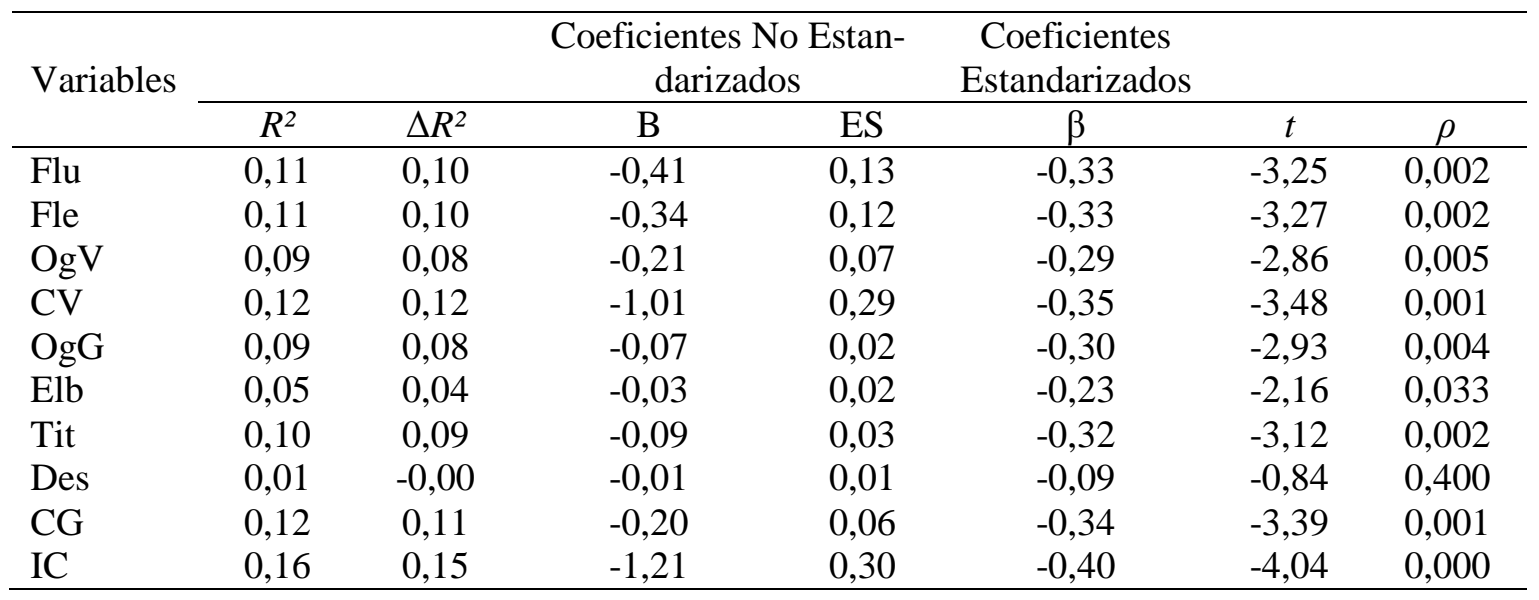

Nota . Flu = Fluidez $;$ Fle = Flexibilidad OgV= Originalidad Verbal $; \mathrm{CV}=$ Creatividad Verbal OgG = Originalidad Gráfica; Elb = Elaboración; Tit = Título; Des = Detalles Especiales; CG = Creatividad Gráfica; IC = Imaginación Creativa. 
Tabla 3. Análisis de regresión lineal simple de la ansiedad rasgo para predecir su relación con los índices de la creatividad verbal y gráfica y la imaginación creativa. $(\mathbf{N}=89)$

\begin{tabular}{lccccccc}
\hline \multirow{2}{*}{ Variables } & \multicolumn{7}{c}{$\begin{array}{c}\text { Coeficientes No Estan- } \\
\text { darizados }\end{array}$} \\
\cline { 2 - 8 } & $R^{2}$ & $\Delta R^{2}$ & B & ES & $\beta$ & $t$ & $\rho$ \\
\hline Flu & 0,24 & 0,24 & $-0,54$ & 0,10 & $-0,49$ & $-5,32$ & 0,000 \\
Fle & 0,24 & 0,24 & $-0,52$ & 0,10 & $-0,49$ & $-5,32$ & 0,000 \\
OgV & 0,23 & 0,22 & $-0,30$ & 0,06 & $-0,48$ & $-5,07$ & 0,000 \\
CV & 0,28 & 0,28 & $-1,36$ & 0,23 & $-0,53$ & $-5,87$ & 0,000 \\
OgG & 0,10 & 0,09 & $-0,07$ & 0,02 & $-0,32$ & $-3,16$ & 0,002 \\
Elb & 0,09 & 0,08 & $-0,04$ & 0,01 & $-0,31$ & $-3,02$ & 0,003 \\
Tit & 0,21 & 0,20 & $-0,11$ & 0,02 & $-0,46$ & $-4,76$ & 0,000 \\
Des & 0,00 & $-0,01$ & $-0,01$ & 0,01 & $-0,06$ & $-0,57$ & 0,568 \\
CG & 0,18 & 0,17 & $-0,23$ & 0,05 & $-0,43$ & $-4,44$ & 0,000 \\
IC & 0,35 & 0,34 & $-1,59$ & 0,23 & $-0,59$ & $-6,80$ & 0,000 \\
\hline
\end{tabular}

Nota . Flu = Fluidez; Fle = Flexibilidad OgV= Originalidad Verbal $; \mathrm{CV}=$ Creatividad Verbal; OgG = Originalidad Gráfica; $\mathrm{Elb}=$ Elaboración; Tit = Título; Des = Detalles Especiales; $\mathrm{CG}=$ Creatividad Gráfica; $\mathrm{IC}=$ Imaginación Creativa.

Los resultados presentados en las Tablas 2 y 3 muestran que tanto la ansiedad estado como la ansiedad rasgo tienen escasa capacidad predictiva para cada uno de los índices que componen la creatividad verbal y gráfica y la imaginación creativa. No obstante, se observa que dicha capacidad es un poco más alta en la ansiedad rasgo para la creatividad verbal $\left[R^{2}=\right.$ 0,$\left.28 ; \Delta R^{2}=0,27 ; t(87)=-5,87 ; p \leq 0,001\right]$, la creatividad gráfica $\left[R^{2}=0,18 ; \Delta R^{2}=0,17 ; t(87)\right.$ $=-4,44 ; p \leq 0,001]$ y la imaginación creativa $\left[R^{2}=0,35 ; \Delta R^{2}=0,34 ; t(87)=-6,80 ; p \leq 0,001\right]$ que en la ansiedad estado si se tiene en cuenta las mismas variables. Estos datos sugieren que tanto la ansiedad estado como la ansiedad rasgo prácticamente no explican los tipos de creatividad ni la imaginación creativa. Además, debido a que la relación entre ambas variables ansiedad y creatividad - es negativa o inversa, podemos señalar que niveles altos de ansiedad proporcionarán predicciones más bajas de creatividad.

En resumen, podría decirse que los estudiantes con altos niveles de ansiedad tuvieron mayores dificultades para ejecutar las tareas de la Prueba de Imaginación Creativa, sobre todo si dicha ansiedad fue más permanente que transitoria.

\section{Discusión y conclusiones}

Los resultados obtenidos en este estudio muestran que existe una asociación negativa y significativa entre los tipos de ansiedad - estado y rasgo- y los tipos de creatividad -verbal y 
gráfica- evaluados, lo que indica que estas dos variables se relacionan mutuamente. Efectivamente, los estudiantes con niveles de ansiedad altos ejecutaron peor las tareas creativas y a la inversa, los creativos reflejaron niveles bajos de ansiedad. Además, el rendimiento creativo fue peor cuando los sujetos presentaban mayor ansiedad rasgo que estado. Las relaciones observadas entre las puntuaciones obtenidas en los tests STAIC y PIC confirman hallazgos de otras investigaciones que también ponen de manifiesto cómo la ansiedad rasgo, es decir, la predisposición propia que tiene un sujeto al responder a diversas tareas influye de forma más determinante que la ansiedad estado que implica una vivencia emocional transitoria caracterizada por la excitación del momento (Byron y Khazanchi, 2011).

Una posible explicación teórica de estos resultados podría ser que la ansiedad rasgo consume recursos cognitivos que las personas creativas necesitan para elaborar un producto creativo (Fuster, 2002). Este consumo distrae y reduce sus capacidades para considerar simultáneamente múltiples ideas, crear imágenes mentales y hacer conexiones dispares. Igualmente, altos niveles de ansiedad rasgo dificultan la actividad de la memoria de trabajo y de la memoria a largo plazo de las personas creativas y reducen su capacidad de atención (Shackman et al., 2006). Tal es así que los sujetos que presentan una ansiedad rasgo continuada tienen déficits a la hora de producir muchas ideas, que pertenezcan a distintas categorías y que sean originales, porque sus memorias de trabajo se ven ocupadas con pensamientos recurrentes sobre el tema que les produce ansiedad. A la hora de recuperar y de establecer ideas remotas entre conceptos, la memoria a largo plazo de dichos sujetos ansiosos se ve constreñida porque se fijan más en datos periféricos que centrales.

Otro aspecto relevante del estudio fue que en la variabilidad total de la creatividad,la ansiedad rasgo mostró una capacidad explicativa más elevada que la ansiedad estado tanto para cada uno de los índices que componen la creatividad verbal (fluidez, flexibilidad y originalidad narrativa) y la creatividad gráfica (originalidad, elaboración, título y detalles especiales) como para la imaginación creativa. Esto pudiera deberse a que los sujetos que presentan mayores niveles de ansiedad rasgo muestran una predisposición más estable, la cual, influiría permanentemente cuando el sujeto realiza tareas que requieren gran esfuerzo y persistencia como son las tareas que tiene mas de una forma de ejecución, es decir, que son creativas. Mientras que la ansiedad estado es peor predictora del nivel de creatividad de los sujetos que la ansiedad rasgo porque al ser una emoción más fugaz se vería, a su vez, más afectada por otro tipo de factores como son por ejemplo: la complejidad de las tareas propuestas o la edad 
de los participantes, tal y como se ha postulado en otras investigaciones (Eysenck et al., 2007; Papousek, Schulter, y Lang, 2009).

En resumen, este estudio apoya las investigaciones que señalan que la ansiedad influye de manera negativa en la ejecución creativa de los sujetos (Matthews, 1986), debido fundamentalmente a que la ansiedad interfiere en los procesos cognitivos responsables de la creatividad. De hecho, los individuos que son propensos a ser más ansiosos dedican una mayor cantidad de recursos cognitivos (por ejemplo: la atención, la memoria de trabajo o la memoria a largo plazo) a atender los síntomas propios de la ansiedad que a resolver las tareas creativas eficientemente. Por ello, sería conveniente que en futuras investigaciones se ampliara el estudio de los procesos cognitivos específicos que pudieran ejercer su influencia en la creatividad o se profundizase en la idea formulada en dos estudios relativamente recientes (Gilhooly, Fioratou, Anthony, y Wynn, 2007; Shackman et al., 2006) que sugieren que los procesos visuales podrían contribuir a la explicación de la asociación negativa entre la ansiedad y la ejecución de tareas de pensamiento divergente. Además, también sería interesante crear programas que entrenen a los sujetos para que aprendan a relajarse lo que favorecería la expresión de su creatividad (Krampen, 1998).

Finalmente, señalar que este estudio adolece de algunas limitaciones como son, el tamaño de la muestra utilizado que no es excesivamente grande lo que implica una generalización prudente de los datos; la selección de la muestra según las posibilidades de acceso a los centros del investigador lo que supone una falta de aleatorización de la misma y, por último, la ausencia de datos procedentes de la aplicación de técnicas de neuroimagen que permitiesen examinar la relación neuroanatómica entre ansiedad y creatividad, es decir, visualizar qué ocurre en el cerebro cuando una persona ansiosa ejecuta tareas creativas verbales o gráficas. No obstante, dicha investigación aporta datos que ayudan a entender mejor las relaciones entre ansiedad y creatividad. 


\section{Referencias}

Ansburg, P. I., y Hill, K. (2003). Creative and analytic thinkers differ in their use of attentional resources. Personality and Individual Differences, 34, 1141-1152.

Artola, R., Ancillo, I., Barraca, J., Mosteiro, P., y Pina, J. (2004). Prueba de Imaginación Creativa. Madrid: TEA Ediciones.

Barron, F. X., y Harrington, D. M. (1981). Creativity, intelligence and personality. Annual Review of Psychology, 32, 439-476.

Byron, K., y Khazanchi, S. (2011). A meta-analytic investigation of the relationship of state and trait anxiety to performance on figural and verbal creative tasks. Personality and Social Psychology Bulletin, 37, 269-283.

Castaneda, A., McCandless, B., y Palermo, D. (1956). The children's form of the Manifest Anxiety Scale. Child Development, 27, 317-326.

Cattell, R. B., y Scheier, I. H. (1961). The meaning and measurement of neuroticism and anxiety. New York: Ronald Press.

Corbalán, J., Martínez, F., Donolo, D., Alonso, C., Tejerina, M., Limiñana, R. (2003). CREA. Inteligencia Creativa: Una medida cognitiva de la creatividad. Madrid: TEA Ediciones.

Elliot, A. J., y McGregor, H. A. (1999). Test anxiety and the hierarchical model of approach and avoidance achievement motivation. Journal of Personality and Social Psychology, $76,628-644$.

Eysenck, M. W., Derakshan, N., Santos, R., y Calvo, M. G. (2007). Anxiety and cognitive performance: Attentional control theory. Emotion, 7, 336-353.

Eysenck, M. W., y Mathews, A. (1987). Trait anxiety and cognition. In H. J. Eysenck \& I. Martin (Eds.), Theoretical foundations of behavior therapy (pp. 197-216). New York, NY: Plenum.

Eysenck, M. W., Derakshan, N., Santos, R., y Calvo, M. G. (2007). Anxiety and cognitive performance: Attentional control theory. Emotion, 7, 336-353.

Fales, C. L., Barch, D. M., Burgess, G. C., Schaefer, A., Mennin, D. S., Gray, J. R., y Braver, T. S. (2008). Anxiety and cognitive efficiency: Differential modulation of transient and sustained neural activity during a working memory task. Cognitive, Affective, y Neuroscience, 8, 239-253.

Flaherty, A. W. (2005). Frontotemporal and dopaminergic control of idea generation and creative drive. Journal of Comparative Neurology, 1, 147-153. 
Fuster, J. M. (2002). Frontal lobe and cognitive development. Journal of Educational Psychology, 66, 67-82.

Garaigordobil, M., y Pérez, J. I. (2002). Análisis predictivo y correlacional de la creatividad gráfica y verbal con otros rasgos de la personalidad infantil. Revista de Psicología General y Aplicada, 55, 373-390.

Gilhooly, K. J., Fioratou, E., Anthony, H., y Wynn, V. (2007). Divergent thinking: Strategies and executive involvement in generating novel uses for familiar objects. British Journal of Psychology, 98, 611-625.

Guilford, J. P. (1984). Varieties of divergent production. Journal Creative Behavior, 18(1), 110.

Heilman, K. M., Nadeau, S. E., y Beversdorf, D. O. (2003). Creative innovation: Possible brain mechanisms. Neurocase, 9, 369-379.

Jones, G., Hanton, S., y Swain, A. B. J. (1994). Intensity and interpretation of anxiety symptoms in elite and nonelite sports performers. Personality and Individual Differences, $17,657-663$

Kashdan T. B. y Fincham, F. D. (2002). Facilitating creativity by regulating curiosity. American Psychologist, 57, 373-374.

Kaufmann, G., y Vosburg, S. K. (1997). "Paradoxical" mood effects on creative problemsolving. Cognition and Emotion, 11, 151-170.

Kinsbourne, M., y Hicks, R. E. (1978). Functional cerebral space: A model for overflow, transfer, and interference effects in human performance. A tutorial. In J. Requin (Ed.), Attention and performance (Vol. 7, pp. 345-362). Hillsdale, NJ: Lawrence Erlbaum.

Krampen, G. (1998). Promotion of creativity (divergent productions) and convergent productions by systematic-relaxation exercises: Empirical evidence from five experimental studies with children, young adults, and elderly. European Journal of Personality, 11, 83-99.

Matthews, G. (1986). The effects of anxiety on intellectual performance:When and why are they found? Journal of Research in Personality, 20, 385-401.

Mumford, M. D., y Gustafson, S. B. (1988). Creativity syndrome: Integration, application, and innovation. Psychological Bulletin, 103, 27-43.

Papousek, I., Schulter, G., y Lang, B. (2009). Effects of emotionally contagious films on changes in hemisphere-specific cognitive performance. Emotion, 9, 510-519.

Richards, A., French, C. C., Keogh, E., y Carter, C. (2000). Test anxiety, inferential reasoning and working memory load. Anxiety, Stress, y Coping, 13, 87-109. 
Scott, G. M., Lonergan, D. C., y Mumford, M. D. (2005). Conceptual combination: Alternative knowledge structures, alternative heuristics. Creativity Research Journal, 4, 91122.

Shackman, A. J., Sarinopoulos, I., Maxwell, J. S., Pizzagalli, D. A., Lavric, A., y Davidson, R. J. (2006). Anxiety selectively disrupts vosuospatial working memory. Emotion, 6, 40-61.

Spielberger, C. D. (1966). The effects of anxiety on complex learning and academic achievement. In: C.D. Spielberger (Ed.), Anxiety and behaviour (pp. 361-398). New York: Academic Press.

Spielberger, D. C. (2001). Cuestionario de Autoevaluación Ansiedad Estado/Rasgo. Madrid: TEA Ediciones.

Sternberg, R. J., y Lubart, T. I. (1993). Creative Giftedness: A multivariate investment approach. Gifted Child Quarterly, 37, 7-15.

Sternberg, R. J., y Lubart, T. I. (1995). Defying the crowd: Cultivating creativity in a culture of conformity. New York: Free Press.

Sternberg, R. J., y Lubart, T. I. (1999). The concepto f creativity: Prospects and paradigms. In R. J. Sternberg (Ed.), Handbook of Creativity (pp. 3-15). Cambridge, England: Cambridge University Press.

Swanner, D. L. (1985). Relationships between musical creativity and selected factors, including personality, motivation, musical aptitude, and cognitive intelligence in third grade children (Unpublished doctoral dissertation). Case Western Reserve University, Cleveland, $\mathrm{OH}$.

Tobias, S. (1985). Test anxiety: Interference, defective skills and cognitive capacity. Educational Psychologist, 20, 135-142.

Tohill, J. M., y Holyoak, K. J. (2000). The impact of anxiety on analogical reasoning. Thinking y Reasoning, 6, 27-40.

Torrance, E. P. (1974). The Torrance test of creative thinking: Norms-technical manual. Bensenvilles IL, ScholasticTesting Service. 